\section{The clinical benefit of genome- wide cfDNA testing cannot be extrapolated from CVS data}

Grati et al. recently published an interesting study in this journal on the clinical outcome of pregnancies with confined placental mosaicism (CPM) detected with chorionic villus sampling (CVS). ${ }^{1}$ The authors extrapolate their CVS data to cell-free DNA (cfDNA) screening and draw the conclusion that genome-wide cfDNA (gw-cfDNA) screening is of minimal benefit since the incidence of adverse pregnancy outcomes in cases of CPM involving rare autosomal aneuploidies (RATs) is low, with the exception of trisomy 16.

We disagree with this conclusion for two main reasons. Firstly, we have concerns about the incomplete data set and erroneous methodology the authors have used to reach this conclusion, and secondly, we disagree with how the CVS results involving a CPM were used to generalize about a lack of clinical utility for gw-cfDNA screening.

Only a very limited part of the original CVS data set was used in the CPM analysis. In the original data set 443 RAT CPM were recorded, with 181 (41\%) included in the study. One or more clinical outcomes were obtained in 124/181 of these CPM cases, which means $72 \%$ of cases (319/443) have been lost to follow-up, which limits conclusions.

A more important concern is that a bias in favor of clinically normal pregnancies is introduced in the already limited data set, partly by including CPM type 2 cases that are undetectable using gw-cfDNA screening, and partly by excluding clinically relevant cases that can be detected. The CVS data set included a large proportion (49/124, or $40 \%)$ of CPM type 2, which represents a mosaic restricted to the mesenchymal core of the chorionic villi. These CPMs are not associated with adverse clinical outcome ${ }^{2}$ and will not be detected by cfDNA testing as the cfDNA originates from the outer cytotrophoblast layer, not the mesenchymal core. On the other hand, CPM cases with ultrasound abnormalities, often seen in cases with a nonmosaic RAT in both cell layers, were excluded. These are mostly of meiotic origin, and are known to have the highest clinical impact. ${ }^{3}$ RATs with abnormal amniocentesis results were also excluded from analysis, which is required to study CPM, but these cases must be included to study the benefits of gw-cfDNA screening.

We question the authors' assertion that the significant relationship between fetal growth restriction (FGR) and pregnancies having CPM is the result of bias. FGR refers to a fetus that has failed to reach its biological growth potential because of placental dysfunction, and not all FGR infants will have a birthweight $<10$ th centile. ${ }^{4}$ FGR was defined heterogeneously between different centers and data on definitions (3rd versus 10th percentiles), biometric parameters, and Doppler are missing. Therefore, no conclusions should have been drawn based on these data. Furthermore, FGR cases were excluded from the neonatal intensive care unit (NICU) admission group but FGR itself is not a reason for NICU admission. This exclusion has introduced an underestimation of the results now reported as being borderline significant.

The authors ignore the rapid technical improvement of gwcfDNA screening methods. Comparing the fetal fraction with the statistical score indicative of a full trisomy is a good predictor for the rate of mosaicism, as has been proven for both the common trisomies and for RATs. ${ }^{5,6}$ Any pregnancy having a RAT result with a high-grade mosaic finding will be at very high risk for a trisomic conception. ${ }^{7}$ These pregnancies are more likely to have pregnancy complications. ${ }^{3,5}$ Further improvements to bioinformatics algorithms may allow for lower grade mosaicisms to be flagged with the option of not reporting them. This would improve test specificity without impacting sensitivity.

Lastly, we want to emphasize that gw-cfDNA screening involves more than the detection of RATs, and will also reveal segmental aneuploidies (SAs) such as duplications, deletions, isochromosomes, and unbalanced translocations. The positive predictive value (PPV) for these SAs to be present in the fetus is much higher than it is for RATs, and can be as high as $50 \%$ in an increased risk population. ${ }^{8}$ Even in the general population the PPV for SAs is around $30 \%$ as observed in Australia (unpublished results) and the Netherlands. ${ }^{9}$ This is much higher than the PPV for RATs, which is around 15\% in a high-risk population and $6 \%$ in the general-risk population (refs. ${ }^{8-10}$ and unpublished data). As RATs and SAs $>10 \mathrm{Mb}$ occur at roughly the same frequency, the SA contribution to the clinical utility of gw-cfDNA testing is expected to be more important compared with the RAT contribution. In essence, a conclusion on the clinical utility of gw-cfDNA screening should never be based on RATs alone.

In conclusion, we appreciate the huge effort the authors have made in putting together these CVS data. However, we do not agree with the extrapolation of the CVS results to gwcfDNA screening. The authors have based their conclusions on the assumption that CVS and cfDNA screening have comparable efficacy, which the authors themselves warned against in a previous publication. ${ }^{10}$ In that study they used the same CVS data set and compared the frequency of true fetal mosaicism (TFM) involving RATs ascertained through CVS and gw-cfDNA. They showed a significantly higher frequency of TFM for RATs ascertained by gw-cfDNA (9.8\%) compared with CVS (3\%) and advised that caution is needed in applying conclusions from CVS analysis to cfDNA testing, and vice 
versa. We agree that conclusions on the clinical impact of $\mathrm{CPM}$ as detected with gw-cfDNA screening can only be drawn from gw-cfDNA screening studies themselves, and should include proper laboratory and clinical follow-up of the majority of cases. Such studies are currently performed, and results will be published in the near future. Preliminary data indicate that gw-cfDNA screening will be clinically relevant. We do agree that currently the additional interpretative complexity of gw-cfDNA screening means this testing should be undertaken in accredited centers with expertise in prenatal cytogenomics, in conjunction with adequate clinical support.

\section{DISCLOSURE}

M.D.P. is employed by the Victorian Clinical Genetics Services (VCGS), which is a not-for-profit provider of gw-cfDNA screening tests and derives income from these tests. VCGS has previously received funding from Illumina Inc. for a gw-cfDNA screening clinical study. M.P.D. declares no personal conflict of interest. The other authors declare no conflicts of interest.

Publisher's note Springer Nature remains neutral with regard to jurisdictional claims in published maps and institutional affiliations.

Erik A. Sistermans, $P h D \mathbb{1 D}^{1}$, Diane Van Opstal, $P h D^{2}$, Mireille N. Bekker, MD, PhD ${ }^{3}$ and Mark D. Pertile, $P h D^{4,5}$

${ }^{1}$ Department of Clinical Genetics, Amsterdam UMC, Vrije Universiteit Amsterdam, Amsterdam, The Netherlands; ${ }^{2}$ Department of Clinical Genetics, Erasmus Medical Center, Rotterdam, The Netherlands; ${ }^{3}$ Department of Obstetrics and Gynaecology, University Medical Center Utrecht, Utrecht University, Utrecht, The Netherlands; ${ }^{4}$ Victorian Clinical Genetics Services, Murdoch Children's Research Institute, Melbourne, VIC, Australia; ${ }^{5}$ Department of Paediatrics, University of Melbourne, Melbourne, VIC, Australia. Correspondence: Erik A. Sistermans (e.sistermans@amsterdamumc.nl)

\section{REFERENCES}

1. Grati FR, Ferreira J, Benn P, et al. Outcomes in pregnancies with a confined placental mosaicism and implications for prenatal screening using cell-free DNA. Genet Med. 2019 Aug 8; https://doi.org/10.1038/ s41436-019-0630-y [Epub ahead of print].

2. Toutain J, Goutte-Gattat D, Horovitz J, Saura R. Confined placental mosaicism revisited: Impact on pregnancy characteristics and outcome. PLoS ONE. 2018;13:e0195905.

3. Robinson WP, Barrett IJ, Bernard L, et al. Meiotic origin of trisomy in confined placental mosaicism is correlated with presence of fetal uniparental disomy, high levels of trisomy in trophoblast, and increased risk of fetal intrauterine growth restriction. Am J Hum Genet. 1997;60: 917-927.

4. McCowan LM, Figueras F, Anderson NH. Evidence-based national guidelines for the management of suspected fetal growth restriction: comparison, consensus, and controversy. Am J Obstet Gynecol. 2018; 218:S855-S868.

5. Pertile MD, Halks-Miller M, Flowers N, et al. Rare autosomal trisomies, revealed by maternal plasma DNA sequencing, suggest increased risk of feto-placental disease. Sci Transl Med. 2017;9:eaan1240.

6. Brison N, Neofytou M, Dehaspe L, et al. Predicting fetoplacental chromosomal mosaicism during non-invasive prenatal testing. Prenat Diagn. 2018;38:258-266.

7. Pertile MD. Genome-wide cell-free DNA-based prenatal testing for rare autosomal trisomies and subchromosomal abnormalities. In: PageChristiaens L, Klein H-G, (eds) Noninvasive prenatal testing (NIPT). London: Academic Press; 2018. pp. 97-123.

8. Van Opstal D, van Maarle MC, Lichtenbelt K, et al. Origin and clinical relevance of chromosomal aberrations other than the common trisomies detected by genome-wide NIPS: results of the TRIDENT study. Genet Med. 2018;20:480-485.

9. van der Meij KRM Sistermans EA, Macville MVE, et al. TRIDENT-2. national implementation of genome-wide non-invasive prenatal testing as a first-tier screening test in the Netherlands. Am J Hum Genet (in press). https://doi.org/10.1016/j.ajhg.2019.10.005.

10. Benn P, Malvestiti F, Grimi B, Maggi F, Simoni G, Grati FR. Rare autosomal trisomies (RATs): a comparison of the detection through cell-free DNA and chorionic villus sampling. Ultrasound Obstet Gynecol. 2019;54: 458-467.

Advance online publication 6 November 2019. doi:10.1038/s41436-019-0689-5 